\title{
A Cost Analysis of Carpal Tunnel Release Surgery Performed Wide Awake versus under Sedation.
}

\author{
Todd H. Alter \\ Thomas Jefferson University \\ William J. Warrender \\ Thomas Jefferson University \\ Frederic E. Liss \\ Thomas Jefferson University
}

Asif M. llyas

Thomas Jefferson University

Follow this and additional works at: https://jdc.jefferson.edu/rothman_institute

Part of the Orthopedics Commons

Let us know how access to this document benefits you

\section{Recommended Citation}

Alter, Todd H.; Warrender, William J.; Liss, Frederic E.; and Ilyas, Asif M., "A Cost Analysis of Carpal Tunnel Release Surgery Performed Wide Awake versus under Sedation." (2018). Rothman Institute Faculty Papers. Paper 112.

https://jdc.jefferson.edu/rothman_institute/112

This Article is brought to you for free and open access by the Jefferson Digital Commons. The Jefferson Digital Commons is a service of Thomas Jefferson University's Center for Teaching and Learning (CTL). The Commons is a showcase for Jefferson books and journals, peer-reviewed scholarly publications, unique historical collections from the University archives, and teaching tools. The Jefferson Digital Commons allows researchers and interested readers anywhere in the world to learn about and keep up to date with Jefferson scholarship. This article has been accepted for inclusion in Rothman Institute Faculty Papers by an authorized administrator of the Jefferson Digital Commons. For more information, please contact: JeffersonDigitalCommons@jefferson.edu. 
1 TITLE:

2 A Cost Analysis of Carpal Tunnel Release Surgery Performed Wide Awake versus under Sedation

4 AUTHORS:

$5 \quad$ (1)Todd H. Alter, B.S. *

6 (2)William J. Warrender, M.D.

7 (3) Frederic E. Liss, MD

8 (4) Asif M. Ilyas, M.D.

9 STUDY LOCATION:

10 The Rothman Institute at the Thomas Jefferson University

11925 Chestnut Street, 5th Floor

12 Philadelphia, PA 19107

\section{DISCLOSURE:}

14 None of the authors received any financial benefit nor had any financial conflicts in the

15 production of this manuscript

16 * CORRESPONDING AUTHOR:

17 Todd Alter

$18 \quad 11341 / 2$ Lombard Street

19 Philadelphia, PA 19147

20 Tel: 609-529-5911

21 E-mail: todd.alter@jefferson.edu

22 PRESENTED AT: American Association for Hand Surgery Annual Meeting. Phoenix,

23 Arizona. January 10-13, 2018.

24 RUNNING HEAD: Economic Analysis Hand Surgery Anesthesia 


\section{$25 \quad$ Financial Disclosures and Products}

26 None of the authors received any financial benefit nor had any financial conflicts in the

27 production of this manuscript. 


\section{Abstract}

29 Background: Hand surgery under local anesthesia only has been utilized more frequently

30 in recent years. The purpose of this study was to compare perioperative time and cost for

31 carpal tunnel release (CTR) performed under local anesthesia (WALANT) only to those

32 performed under intravenous sedation (MAC).

33 Methods: A retrospective comparison of intra-operative (OR) surgical time and post-

34 operative (PACU) time for consecutive CTR procedures performed under both MAC and

35 WALANT was undertaken. All operations were performed by the same surgeon using the

36 same mini-open surgical technique. A cost analysis was performed via standardized

37 anesthesia billing based on base units, time, and conversion rates.

38 Results: There were no significant differences between the two groups in terms of total

39 OR time, 28 minutes in the MAC group versus 26 minutes in the WALANT group.

40 PACU times were significantly longer in the MAC group (84 minutes) compared to the

41 WALANT group (7 minutes). Depending on conversion rates used, a total of \$139-\$432

42 was saved in each case done with WALANT by not using anesthesia services. In

43 addition, a range of $\$ 1,320-\$ 1,613$ was saved for the full episode of care including

44 anesthesia costs, OR time, and PACU time for each patient undergoing WALANT CTR.

45 Conclusions: CTR surgery performed with the WALANT technique offers significant

46 reduction in cost utilization of anesthesia and PACU resources. 


\section{$47 \quad$ Introduction}

48 Hand surgery performed under local anesthesia only without a tourniquet, also

49 called "Wide Awake Local Anesthesia No Tourniquet" (WALANT) surgery, is a

50 technique that is experiencing growing interest and utilization. [8,13-18,22,24] In

51 WALANT surgery, surgical pain is controlled through an injection of a local anesthetic.

52 The patient does not receive an intravenous or general anesthetic agent, thus eliminating

53 the need for an anesthesia provider. There is minimal bleeding because the local

54 anesthetic is supplemented with epinephrine, which limits bleeding within the operative

55 field and negates the need and discomfort of a tourniquet. While there exists a widely

56 accepted belief that use of epinephrine in distal extremities can cause finger necrosis,

57 recent studies have demonstrated both the safety and efficacy of epinephrine utilization in

58 the hand.[8,11,13-18,22,24,26-27]

59 Advocates for WALANT claim that this anesthetic method is safer as it eliminates

60 standard anesthetic risks; is more convenient for the patient as it foregoes the need for

61 pre-operative diagnostic testing, eliminates fasting, eliminates the need for a driver,

62 avoids the discomfort of having an IV placed, foregoes anesthesia induction time in the

63 operating room, and minimizes post-anesthesia care unit (PACU) recovery time. Another

64 possible benefit of WALANT surgery is financial.[8,14-15] There are several potential

65 cost savings from utilizing the WALANT technique rather than intravenous sedation with

66 local (MAC). Areas worthy of economic comparison include the elimination of pre-

67 operative testing (blood work, EKG and physician consultation for clearance for

68 anesthesia), reducing and/or possibly eliminating the cost of an anesthesia provider and 
69 the cost savings introduced by reducing the time patients need to spend in the operating

$70 \operatorname{room}(\mathrm{OR})$ and PACU.

71 The purpose of this study was to perform an economic analysis of hand surgery

72 utilizing a carpal tunnel release (CTR) surgery model, by comparing the facility costs of

73 CTR surgery performed under WALANT versus those performed under MAC. The

74 hypothesis was made that WALANT surgery would result in decreased hospital cost as

75 compared to MAC surgery.

$77 \quad$ Materials and Methods

78 After receiving Institutional Review Board approval for retrospective review and

79 analysis of patient records, a comparison of all CTR surgery performed between 2012-

802015 were reviewed. All surgeries were performed by one fellowship-trained hand

81 surgeon, at our outpatient surgical center. All procedures followed were in accordance

82 with the ethical standards of the responsible institutional committee on human

83 experimentation. The surgeon transitioned from performing all CTR surgeries under

84 MAC to WALANT at the end of 2013. Therefore, consecutive CTR surgery performed

85 between 2012-2013 with MAC were available for comparison with CTR cases in 2014-

8615 performed consecutively with the WALANT technique. Inclusion criteria were all

87 "mini-open" CTR surgeries performed alone without concomitant procedures. Data

88 points collected included: total OR time, surgical time, and PACU time, which were

89 retrieved from the anesthesia record and nursing documentation. Anesthetic

90 complications and re-operations were also recorded. These data points were compared

91 and statistically analyzed using two-tailed T-tests. 
93 Both MAC and WALANT patients physically walked themselves into the

94 operating room. Patients were positioned supine with their operative arm extended onto a

95 hand table. A non-sterile tourniquet on the upper arm was applied to those undergoing

96 MAC, while no tourniquet was applied in the WALANT group. Both groups underwent

97 similar prepping and draping. There was no change in the surgical scrub, preparation, and 98 draping during the study period.

\section{Induction of Anesthesia}

100 After prepping and draping the surgical site and after induction of anesthesia for

101 the MAC cases, but prior to initiating surgery, 10cc of $1 \%$ lidocaine plain (without

102 epinephrine), was injected into the surgical site. After injection but prior to making

103 incision, the MAC group underwent Esmarch exsanguination the limb followed by

104 insufflation of the tourniquet to $250 \mathrm{mmHg}$.

105 For patients undergoing WALANT, 9cc of 1\% lidocaine with 1:100,000

106 epinephrine and $1 \mathrm{cc}$ of sodium bicarbonate were mixed. A total of $10 \mathrm{cc}$ of this mixture

107 was injected into the surgical site upon entry into the operating room but prior to

108 prepping and draping the patient.[19] Although it has been recommended to pre-inject the

109 surgical site 20-30 minutes prior to injection in the pre-operative unit, it has been the

110 practice of the senior author (AMI) to inject in the operating room prior to prepping and

111 draping the patient with a negligible difference in bleeding and still no need for a

112 tourniquet. An additional 10cc of 1\% lidocaine with 1:100,000 epinephrine was available

113 on the field for additional injection, as needed. Phentolamine, a reversal agent for the 
114 vasoconstrictive effects of epinephrine, was available at all times in the surgical center

115 but never needed to be utilized in any case.

117 The identical "mini-open" CTR surgical technique was utilized for both the MAC

118 and WALANT cases. A $2 \mathrm{~cm}$ longitudinal incision was placed at the base of the volar

119 hand in line with the third web space. The superficial palmar fascia was cut in line with

120 the skin incision. The transverse carpal ligament was identified and released

121 longitudinally until complete decompression of the median nerve was confirmed. The

122 skin incisions were closed with three horizontal mattress 4-0 nylon sutures followed by

123 application of soft sterile dressing.

\section{Cost Analysis}

125 Anesthesia costs are calculated based on a base unit value assigned to the

126 procedure based on its complexity added to the number of 15 -minute time units the

127 provider spends multiplied by the provider's charge per unit (i.e. conversion factor). The

128 anesthesia clock starts when anesthesia personnel begin to prepare the patient for

129 anesthesia care, and ends when the patient is safely placed in post-anesthesia supervision

130 and anesthesia personnel are no longer in personal attendance. We used the 2017 Centers

131 for Medicare and Medicaid Services (CMS) conversion rate of $\$ 23.14$ for our

132 institution's metropolitan area.[6] The anesthesia CPT code 01810 was used to determine

1333 base units are applied to basic nerve decompression surgery of the hand. For each case

134 done under WALANT a total of $\$ 138.84$ was saved by not using anesthesia services.

135 Please see below for calculation.

136 [Base unit + Time (units) $] \times$ Conversion factor $=$ Anesthesia charge

$137 \quad[3$ units +3 units $] \times \quad \$ 23.14 /$ unit $=\$ 138.84 /$ case 
140 separately billable to the Medicare Program. To evaluate the potential cost savings to the

141 hospital when WALANT surgery was used for CTR, we used $\$ 12.16$ /minute that a

142 patient is in the PACU based on a 2015 study that evaluated detailed list of direct and

143 indirect costs needed rather than amount billed. We felt this would provide the best

144 estimate of potential cost savings to the hospital.[21]

145 Finally, to estimate standard costs for items associated with a routine pre-

146 operative testing associated with anesthesia we used figures publicly available from

147 healthcarebluebook.com. This database used averages of actual amounts paid by

148 insurance companies, including CMS, for common medical tests and services. The

149 standard preoperative testing consists of a history and physical, chest radiographs,

150 electrocardiogram, and standard bloodwork in the form of a complete blood count and

151 basic metabolic panel.

\section{$153 \underline{\text { Results }}$}

154 A total of 190 patients met the inclusion criteria; 136 underwent sedation with

155 MAC and 54 patients with WALANT surgery. The average ages were $59 \pm 14$ and $62 \pm$

15612 for MAC and WALANT groups, respectively. There were 86 females in the MAC

157 group (63\%) and 24 in the WALANT group (44\%). There were no anesthetic

158 complications or re-operations in either group.

159 As defined by the in-room and out-room time, patients in the MAC group had an

160 average total OR time of $28 \pm 5.5$ minutes, while the WALANT group averaged $26 \pm 6.7$

161 minutes $(\mathrm{p}=0.052)$. 
The surgical time, as defined by the documented procedure start and end time,

163 averaged $9.7 \pm 2.2$ minutes in the MAC group while the WALANT group averaged $10 \pm$

1642.3 minutes $(\mathrm{p}=0.41)$.

165 Post-operatively, patients in the MAC group spent an average of $84 \pm 29$ minutes

166 in the recovery room prior to discharge, compared to $7 \pm 2$ minutes in the WALANT

167 group $(\mathrm{p}<0.05)($ Table 1$)$.

168 As anesthesia reimbursement and individual patient insurance contracts differ, we

169 used standard CMS reimbursement rates for anesthesia to determine cost differences. We

170 estimated that each case performed under MAC had excess charges secondary to

171 anesthesia reimbursement of approximately $\$ 138.84$ (See Methods for calculation). At a

172 rate of $\$ 12.16 /$ minute,[21] with an average 84 minutes in the PACU, MAC cases cost an

173 additional $\$ 1,021.44$ to the hospital. Furthermore, we estimate that patients scheduled for

174 MAC had standard preoperative medical clearance and testing consisting of a history and

175 physical (established patient 25 min visit: \$117), chest radiographs (\$47),

176 electrocardiogram (\$22), and standard bloodwork in the form of a complete blood count

177 (\$21) and basic metabolic panel (\$28).[9] The cost for these preoperative expenditures is

$178 \$ 235$ per patient. Additionally, a pneumatic tourniquet cuff and $10 \mathrm{cc}$ of $1 \%$ lidocaine

179 without epinephrine were used in each case, costing \$10[2] and \$4.[1]

180 Patients undergoing CTR under WALANT spent an average of 7 minutes in the

181 PACU and thus assumed a cost of $\$ 85.12$ (\$12.16/minute). In each WALANT case, 20cc

182 of $1 \%$ lidocaine with 1:100,000 epinephrine was used, costing an additional \$4.[1] The

183 WALANT patients assumed a $\$ 0$ cost for all of the remaining preoperative and

184 postoperative expenditures. 
186 lidocaine costs, and PACU costs, each patient performed under WALANT saved the

187 healthcare system an average of $\$ 1,320.16$ (Table 2).

$189 \underline{\text { Discussion }}$

190 Carpal Tunnel Syndrome is a common hand condition that is often treated with a

191 CTR when surgery is indicated.[12] Perioperative anesthesia with sedation (MAC) or

192 general anesthesia has traditionally been used for routine hand surgical procedures such

193 as CTR. Recently, advances in WALANT technique has given surgeons and patients an

194 additional method of administering anesthesia for routine hand surgical procedures such

195 as CTR, thereby forgoing the need for anesthesia staff involvement and PACU recovery

196 time.[14-15,18]

197 In our analysis of WALANT hand surgery performed in the treatment of carpal

198 tunnel release surgery, we found no significant difference in the length of the procedure

199 or in the total time spent in the operating room compared to the use of MAC. We

200 speculate that the time spent by anesthesia to sedate the patient in the MAC group was

201 matched by the time spent injecting local anesthetics by the surgeon in the WALANT

202 group. In addition, the similarity in length of procedure indicates that the epinephrine

203 used in the WALANT group was as effective in controlling bleeding as the tourniquet in

204 the MAC group.

205 Pre-operative nursing time was not measured. In general, patients in the MAC

206 group would be expected to spend greater time in pre-op for IV placement and anesthesia

207 evaluation, discussion, and consenting. Alternatively, patients in the WALANT group did 
208 not require IV or anesthesia staff involvement. Conceivably, surgeons may request

209 patients to present early to the pre-operative unit for injection of the surgical site as it is

210 recommended to give the epinephrine 20-30 minutes to maximize the vasoconstrictive

211 effect.[19] However, it is the practice of the senior author (AMI) to inject immediately

212 pre-operatively in the operating room, prior to prepping the limb, without any increased

213 issue of bleeding or need for a tourniquet. As such, for study purposes, we ignored the

214 pre-operative time as that may be variable among surgeons. Regardless, had we studied

215 this in our study we would have only found more time and cost in the MAC group.

216 Post-anesthesia care unit (PACU) times were significantly shorter in the

217 WALANT group ( $7 \pm 2$ minutes) compared to the MAC group ( $84 \pm 29$ minutes).

218 Because patients in the WALANT group do not receive systemic anesthetic agents, they

219 do not require time to recover from their effects. An additional advantage for patients in

220 the WALANT group is that they are allowed to drive themselves home following the

221 procedure, and they therefore do not have to arrange for a ride.

222 The cost savings for each patient we found was $\$ 1,320.16$. This is an intentional,

223 gross underestimation of the potential cost savings possible for WALANT CTR. Our

224 anesthesia cost analysis was based on Medicare reimbursement schedules with no

225 representation of the reimbursements of private insurers. We used the $2017 \mathrm{CMS}$

226 conversion rate of $\$ 23.14$ for our institution's metropolitan area. Based on the 2015

227 American Society for Anesthesiologists (ASA) commercial conversion factor survey

228 results, the national average conversion factor was \$71.92.[23] If we used this national

229 average conversion rate, our cost savings would increase to $\$ 431.52$ for anesthesia

230 services and \$1,612.84 total per patient. Some premium insurance plans reviewed pay as 
231 much as $\$ 140 /$ /mit in major metropolitan areas.[23] In addition, because PACU cost is

232 not billed separately to insurance it is difficult to estimate the true cost. For this reason,

233 we used the detailed cost analysis performed previously by Raft et al.[21] Because it is

234 not based on billing charges, we feel this is the best estimate of actual cost (direct and

235 indirect) saved by the hospital but actually underestimates the final amount billed to the

236 insurance company.

237 Codding et al. performed an economic analysis in which 78 consecutive cases of

238 single trigger finger release surgery with MAC (31) were compared to those with

239 WALANT (47).[7] Patients in the MAC group experienced an average OR time and

240 surgical time of 27.2 and 10.2 minutes, respectively. Similarly, patients in the WALANT

241 group experienced an average OR time and surgical time of 25.2 and 10.4 minutes,

242 respectively. Average recovery room time was 72.3 and 30.2 minutes in the MAC and

243 WALANT groups, respectively. This study reported an average savings of $\$ 105$

244 secondary to anesthesia reimbursement in MAC cases. However, while the average OR,

245 surgical, and recovery room times were similar to our study, there was little detail on

246 objective cost data (pre-op clearance, PACU, bloodwork, etc.) outside of anesthesia

247 reimbursement, rendering the estimate of cost savings far less than reality.

248 Anesthesia is also associated with increased rates of nausea and vomiting.

249 Twenty-six percent of patients require additional treatment in the PACU, and $40 \%$ of

250 patients require additional treatment for post-operative nausea and vomiting (PONV)

251 following discharge.[3-4] The cost of rescue treatment for PONV has been estimated at a

252 minimum of $\$ 283$.[10] While we did not record PONV, this is an issue that can result in

253 the need ambulance transfer to a hospital costing $\$ 300$ - $\$ 900$ and result in an admission 
254 costing $\$ 1,200$ to more than $\$ 2,400$ per day. Additionally, an economic impact would

255 also be seen in patients with obstructive sleep apnea (OSA). Studies show that $22-39 \%$ of

256 all surgical patients are at high risk for OSA. $80 \%$ of these patients are undiagnosed.[28]

257 During recovery, residual anesthetics increase the number and duration of sleep apnea

258 episodes but inhibit arousals which would normally occur during such episodes. For this

259 reason, the guidelines from the ASA for perioperative care of OSA patients suggest

260 patients should stay in recovery for an extended period after the last episode

261 (desaturation, reintubation, hypoxia, etc.). Eliminating these possible anesthetic

262 complications completely further reduces budget for PACU time and cost. Finally, we

263 evaluated the basic costs for a pre-operative visit and testing associated with clearance for

264 anesthesia. Additional costs would be incurred for patients required to see a cardiologist

265 or other specialist or if any further testing (echocardiogram, stress test, advanced lab

266 work) was needed prior to surgery.

267 While there have been reports that use of epinephrine in distal extremities can

268 cause finger necrosis,[29] these events appear to be extremely rare and recent studies

269 have demonstrated both the safety and efficacy of epinephrine utilization in the hand.

$270 \quad[8,11,13-18,22,24,26-27]$ In the senior author's personal experience of performing over

2712000 cases under WALANT, there have been zero cases of digital ischemia or need for

272 reversal. Nonetheless, it is good practice to keep phentolamine, a reversal agent for the

273 vasoconstrictive effects of epinephrine, available at the surgical center where WALANT

274 surgery is being performed.[20] There is an associated cost in ensuring that there is

275 phentolamine (\$35 for $50 \mathrm{mg}$ )[5] available that has not expired. However, as the use of

276 phentolamine is extremely rare, it would have a negligible cost when spread out among 
277 all WALANT cases occurring over the course of its shelf life. In addition, patients should

278 be advised that they may feel jittery or shaky following injection but that this typically

279 dissipates in 15-20 minutes.[14] This potential side effect requires no additional treatment

280 and thus does not affect costs.

281 The purpose of the study was designed to investigate perioperative times and

282 perform an economic analysis and comparison of CTR performed under MAC vs.

283 WALANT. Therefore, no outcome measures, patient satisfaction scores, or follow-up

284 data were collected. Several studies have, however, investigated such data in patients

285 undergoing WALANT carpal tunnel release surgery. Davidson et al. found that $93 \%$ of

286 patients who underwent WALANT surgery would choose it again in subsequent

287 surgeries. In addition, it was found that patients' intraoperative anxiety for WALANT

288 surgery was not significantly different than their preoperative anxiety. However, patients

289 who underwent WALANT surgery had significantly less preoperative anxiety than

290 patients who underwent sedation.[8] Teo et al. similarly found that $86 \%$ of patients who

291 underwent WALANT surgery would choose it again in subsequent surgeries.

292 Additionally, 91\% reported that the operation was less painful or comparable with a

293 procedure at the dentist.[25]

294 Our study has some limitations. First, it is a retrospective study that is based

295 solely on the experiences of a single surgeon at a single institution. Multi-center,

296 prospective, randomized trials could build upon our data and provide further insight into

297 use of the WALANT method. As previously noted, this study was not designed to

298 investigate outcomes or patient satisfaction and thus lacks any clinical data. In addition,

299 the costs assumed by hospitals, patients, and insurance companies vary significantly and 
300 often are difficult to dissect on an item-by-item basis. This study aims to address costs to

301 the hospital, but payer reimbursements often were used in their place when sufficient data

302 was unavailable, which may affect the accuracy of our cost estimates.

303 In conclusion, patients who underwent carpal tunnel release surgery under

304 WALANT demonstrated similar time in the operating room and similar surgical time

305 from incision to closure compared to sedation with MAC. Patients in the WALANT

306 group also spent significantly less time in the PACU post-operatively, saving

307 approximately $\$ 1,320.16$ per patient, which certainly underestimates the total savings.

308 Avoiding use of anesthesia services for high volume procedures like carpal tunnel release

309 surgery may result in significant systemic annual savings to payers and hospitals. These

310 savings may be desirable with the growth of bundling and episode-based payments to

311 patients, facilities, and surgeons. 


\section{$312 \underline{\text { References }}$}

314 1. ACE Surgical Supply Co., Inc. http://www.acesurgical.com/. Accessed May 1, 2018.

315 2. Alibaba. https://www.alibaba.com/. Accessed May 1, 2018.

316 3. Apfel CC, Korttila K, Abdalla M, et al. A factorial trial of six interventions for the

317 prevention of postoperative nausea and vomiting. N Engl J Med. 2004;350(24):2441-

$318 \quad 2451$.

319 4. Carroll NV, Miederhoff P, Cox FM, Hirsch JD. Postoperative nausea and vomiting

320 after discharge from outpatient surgery centers. Anesth Analg. 1995;80(5):903-909.

321 5. Cayman Chemical. https://www.caymanchem.com. Accessed May 1, 2018.

322 6. Centers for Medicare and Medicaid Services. (2016, January 12). Retrieved June 19,

323 2017, from https://www.cms.gov/Center/Provider-Type/Anesthesiologists-

$324 \quad$ Center.html

325 7. Codding JL, Bhat SB, Ilyas AM. An Economic Analysis of MAC Versus WALANT:

326 A Trigger Finger Release Surgery Case Study. Hand (N Y). 2017;12(4):348-351.

327 8. Davison PG, Cobb T, Lalonde DH. The patient's perspective on carpal tunnel surgery

328 related to the type of anesthesia: a prospective cohort study. Hand (NY). 2013

$329 \quad$ Mar;8(1):47-53.

330 9. Healthcare Bluebook. (n.d.). Retrieved June 19, 2017, from

$331 \quad$ http://www.healthcarebluebook.com/

332 10. Hill RP, Lubarsky DA, Phillips-bute B, et al. Cost-effectiveness of prophylactic

333 antiemetic therapy with ondansetron, droperidol, or placebo. Anesthesiology.

$334 \quad 2000 ; 92(4): 958-967$. 
335 11. Ketonis C, Ilyas AM, Liss F. Pain Management Strategies in Hand

336 Surgery. Orthopedic Clinics of North America. 2015 Mar;46(3), 399-408.

337 12. Kim PT, Lee HJ, Kim TG, Jeon IH. Current approaches for carpal tunnel syndrome.

338 Clin Orthop Surg. 2014;6(3):253-257.

339 13. Lalonde D, Eaton C, Amadio P, Jupiter J. Wide-awake hand and wrist surgery: a new

340 horizon in outpatient surgery. Instr Course Lect. 2015; 65:249-259.

341 14. Lalonde D, Martin A. Epinephrine in local anesthesia in finger and hand surgery: the

342 case for wide-awake anesthesia. J Am Acad Ortho Surg. 2013 Aug;21(8):443-447.

343 15. Lalonde D, Martin A. Tumescent local anesthesia for hand surgery: improved results,

344 cost effectiveness, and wide-awake patent satisfaction. Arch Plast Surg. 2014

$345 \quad$ Jul;41(4):312-316.

346 16. Lalonde D. Minimally invasive anesthesia in wide awake hand surgery. Hand Clin.

$3472014 \mathrm{Feb} ; 30(1): 1-6$.

348 17. Lalonde DH. Wide-awake extensor indices proprius to extensor pollicis longus tendon

349 transfer. J Hand Surg Am. 2014 Nov;39(11):2297-2299.

350 18. Lalonde DH, Wong A. Dosage of local anesthesia in wide awake hand surgery. $J$

$351 \quad$ Hand Surg Am. 2013 Oct;38(10):2025-2028.

352 19. Mckee DE, Lalonde DH, Thoma A, Dickson L. Achieving the optimal epinephrine

353 effect in wide awake hand surgery using local anesthesia without a tourniquet. Hand

$354 \quad$ (N Y). 2015;10(4):613-615.

355 20. Nodwell T, Lalonde DH: How long does it take phentolamine to reverse adrenaline-

356 induced vasoconstriction in the finger and hand? A prospective randomized blinded 
study. The Dalhousie project experimental phase. Canadian Journal of Plastic

$358 \quad$ Surgery 2003;11(4):187-190.

359 21. Raft J, Millet F, Meistelman C. Example of cost calculations for an operating room

360 and a post-anaesthesia care unit. Anaesth Crit Care Pain Med. 2015;34(4):211-215.

361 22. Ruxasagulwong S, Kraisarin J, Sananpanick K. Wide awake technique versus local

362 anesthesia with tourniquet application for minor orthopedic hand surgery: a

363 prospective clinical trial. J Med Assoc Thai. 2015 Jan; 98(1):106-110.

364 23. Stead SW, Merrick SK. ASA Survey Results for Commercial Fees Paid for

365 Anesthesia Services - 2015. ASA Monitor. 2015;79(10):48-54.

366 24. Tang JB. Wide-Awake Primary Flexor Tendon Repair, Tenolysis, and Tendon

367 Transfer. Clin Orthop Surg. 2015;7(3):275-281.

368 25. Teo I, Lam W, Muthayya P, Steele K, Alexander S, Miller G. Patients' perspective of

369 wide-awake hand surgery- 100 consecutive cases. J Hand Surg Eur. 2013 Nov;

$370 \quad 38(9): 992-999$.

371 26. Vinycomb TI, Sahhar LJ. Comparison of local anesthetics for digital nerve blocks: a

372 systematic review. J Hand Surg Am. 2014 Apr;39(4):744-751.

373 27. Youha AS, Lalonde DH. Update/Review: changing of use of local anesthesia in the

374 hand. Plast Reconstr Surg Glob Open. 2014 Jun 6;2(5):e150.

375 28. Young T, Peppard PE, Gottlieb DJ. Epidemiology of obstructive sleep apnea: a

376 population health perspective. Am J Respir Crit Care Med. 2002;165(9):1217-1239.

377 29. Zhang JX, Gray J, Lalonde DH, Carr N. Digital Necrosis After Lidocaine and

378 Epinephrine Injection in the Flexor Tendon Sheath Without Phentolamine Rescue. $J$

$379 \quad$ Hand Surg Am. 2017;42(2):e119-e123. 
$380 \quad$ Legend

381 Table 1 - Comparison of time variables for MAC vs. WALANT.

382 Table 2 - Cost comparison for MAC vs. WALANT. 